Check for updates

Cite this: RSC Adv., 2019, 9, 27659

Received 23rd July 2019

Accepted 29th August 2019

DOI: 10.1039/c9ra05689k

rsc.li/rsc-advances

\title{
Catalysis with carbon nanoparticles
}

\author{
Caterina Testa, ${ }^{a}$ Agatino Zammataro, (D) a Andrea Pappalardo (D) ab \\ and Giuseppe Trusso Sfrazzetto (iD *ab
}

Carbon nanoparticles (CNPs) represent a recent class of nanomaterials, based on carbon $\mathrm{sp}^{2}$ atoms in the inner core. These new nano-dots cover a wide range of application fields: analytical, sensing and biosensing, bioimaging, theranostic, and molecular communication. However, their use as nanocatalysts is relatively new. Although CNPs can be easily synthesized and obtained in good amounts, few reports on their catalytic applications have been reported. This minireview collects the use of these nanoparticles as catalysts highlighting the improvements with respect to the classic catalytic systems. In particular, due to their unique optical and electrical properties, and due to the possibility to cover the external shell with a wide variety of functional groups, CNPs have found catalytic applications in three main classes of reactions: (i) photocatalysis, (ii) acid-base catalysis and (iii) electro catalysis.

\section{Introduction}

Carbon nanostructures represent a wide class of organic compounds having different structures, including well-known fullerene, graphene, carbon nanotubes, and also other lessexplored carbon nanoforms, such as carbon nanoparticles (CNPs) and nanodiamonds. These nanostructures, characterized by the predominant presence of $\mathrm{sp}^{2}$-bonded carbon atoms, can be classified based on the number of dimensions. For example, although their dimensions are at least $1 \mathrm{~nm}$, pristine fullerene and carbon nanoparticles can be classified as zerodimensional compounds, carbon nanotubes (carbon material that can be further classified into single-walled carbon nanotubes, SWNT, and multi-walled carbon nanotubes, MWNT) as one-dimensional and graphene as two-dimensional (Fig. 1). ${ }^{1}$

Carbon Nanoparticles (CNPs) are a new class of zerodimensional carbon-based nanomaterials having a diameter range of 3-10 $\mathrm{nm}$. Their internal structure is based on graphite sheets assembled with each other, leading to a quasi-spherical nanoparticle. ${ }^{2}$ The external shell can be decorated with a wide range of functional groups, depending on the preparation methodology of $\mathrm{CNPs}^{3}$ and also on the covalent functionalization with the appropriate organic molecule. Preparation of CNPs is cheap and simple and can be performed by different protocols, such as microwave irradiation, hydrothermal carbonization, pyrolysis, reverse micelles and converting from carboxyl to amine groups. ${ }^{4}$

Following this first strategy, the starting organic carbon source will define the composition of the external shell (Scheme 1a), on the other hand, the external shell of CNPs can be further

${ }^{a}$ Department of Chemical Sciences, University of Catania, Viale Andrea Doria 6, Catania, Italy, 95125. E-mail: giuseppe.trusso@unict.it

${ }^{b}$ INSTM Udr of Catania, Viale Andrea Doria 6, Catania, Italy, 95125 functionalised by covalent reaction between appropriate coupling agent (Scheme 1b), leading to a stable covalent bond.

However, the one-pot synthesis leads to CNPs which do not show the properties achieved by the covalent functionalization, in term of reactivity and sensing properties.

During this decades, the publication number related to carbon nanoparticles increased, from 5 in the 2010, to 852 in the 2018, highlighting the growing interest to this "new" class of nanoparticles.

CNPs show peculiar optical properties. In particular, the typical UV-Vis spectrum presents a broad absorption band in the UV region with a tail in the visible region (up to $600 \mathrm{~nm}$ ), attributed to $\pi-\pi^{*}$ and $n-\pi^{*}$ transitions, relative to $\mathrm{C}=\mathrm{C}$ and $\mathrm{C}=\mathrm{O}$ double bond, respectively. The emission profile depends by the excitation wavelength: moving from lower to higher

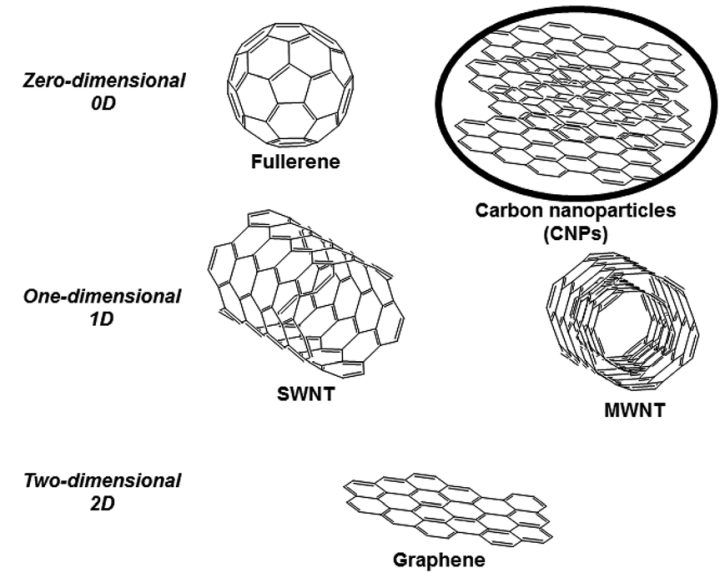

Fig. 1 Most common carbon nanostructures. 
a)

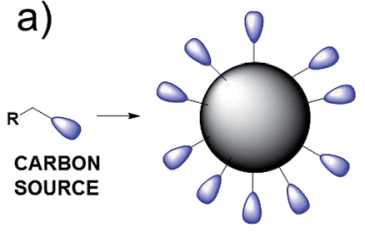

b)

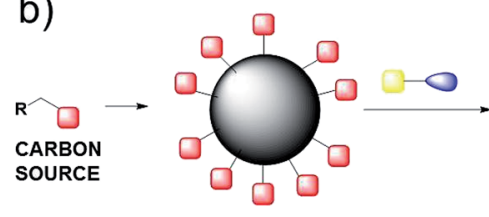

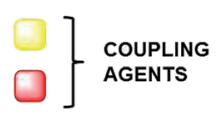

FUNCTIONAL GROUP

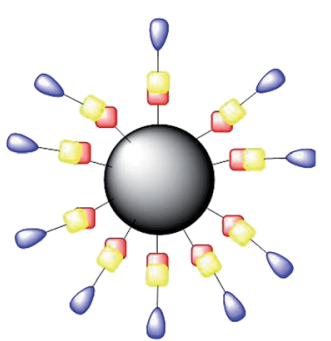

Scheme 1 (a) One-pot synthesis of CNPs; (b) covalent functionalization of the external shell.

excitation wavelengths, also the emission band shifts from lower to higher nanometers.

Carbon nanoparticles find applications in several fields, such as analytical, ${ }^{5}$ biosensing, ${ }^{6}$ bioimaging, ${ }^{7}$ theranostic, ${ }^{8}$ and molecular communication. ${ }^{9,10}$ However, to date, few examples of CNPs used as catalysts have been reported. In this context, the well-known increased catalytic properties of a catalyst covalent immobilized onto a solid surface (heterogeneous catalysis $)^{11-13}$ are also founded with carbon nanoparticles as nanocatalysts.

\section{Photocatalysts}

A photocatalyst is a material which absorbs the energy of the light leading to a higher energy level, and releases such energy to a one or more substrates (reagents) catalysing a specific reaction. The use of CNPs as photosensitizers displays several advantages, such as abundance, nontoxicity, stability and sustainability. In this context, Uthirakumar and co-workers synthesized CNPs doped with nitrogen and ZnO, characterized by SEM, TEM, Raman, X-ray and UV-Vis spectroscopy. ${ }^{14}$ These nanoparticles show a broad absorption band extended to $600 \mathrm{~nm}$, with an average particle size of $c a .25 \mathrm{~nm}$, with the presence of an external shell of $0.26 \mathrm{~nm}$ due to the presence of ZnO. These nanoparticles have been used as catalysts for the degradation of three different industrial dyes by using the daylight irradiation: malachite green, methylene blue and fluorescein. For example, CNPs doped with $\mathrm{ZnO}$ show higher degradation efficiency of malachite green dye (100\%), if compared to simple $\mathrm{Zn}-\mathrm{O}$ doped with nitrogen (60\%), demonstrating the positive role of the nanocatalyst in the dye degradation. In the case of methylene blue, nanocatalyst degraded completely the dye in 45 minutes, while the control experiments show $82 \%$ of degradation in 60 minutes. With fluorescein, a $95 \%$ of degradation occurs in 15 minutes $(100 \%$ in 30 minutes) with the synthesised nanocatalyst, while in the control experiments the $92 \%$ of degradation requires 60 minutes. Furthermore, the nanocatalysts have been reused 4 times, preserving the efficiency.

Reisner and co-workers employed the photochemical properties of CNPs with a catalyst containing nickel for the $\mathrm{H}_{2}$ production in aqueous solution by using solar light. ${ }^{15}$ The nanocatalyst has been prepared by thermolysis of citric acid in an one-pot reaction. XPS measurements suggest that the surface of these CNPs is covered by carboxylic groups. The morphology of the obtained nanoparticles have been characterized by TEM analysis, showing a diameter of $c a .6 .8 \mathrm{~nm}$, with the presence of both graphitic and amorphous carbon atoms. Optical properties were evaluated by UV-Vis and emission spectroscopy, confirming the typical behaviour of CNPs above reported.

These nanoparticles catalyse the hydrogen production receiving the UV-Vis solar radiation and transfering the energy to the Ni-catalysts, improving the photostability of the system (Fig. 2). The best hydrogen production performance was 398 $\mu \mathrm{mol} \mathrm{H} \mathrm{H}_{2}\left(\mathrm{~g}_{\mathrm{CNPs}}\right)^{-1} \mathrm{~h}^{-1}$, with a TON (Turn Over Number) value of $41 \mathrm{~h}^{-1}$. CNPs are highly lasting light-absorbing material for photocatalytic schemes, not restricted by cost or toxicity, moreover the use of CNPs as photosensitizers displays several advantages, such as abundance, nontoxicity, stability, and sustainability. In the absence of carbon nanoparticles, evolution of hydrogen does not occur.

N-doped and amino terminated-CNPs, synthesized by microwave-assisted hydrothermal protocol, have been used by Moretto and co-workers to realize composite materials, based on common polymers like polyamide, polyurea-urethane, polyester, and polymethylmetacrylate, in order to obtain useful materials, such as fibers, transparent sheets, and bulky forms. ${ }^{16}$

These CNPs show a diameter of $c a .5 \mathrm{~nm}$, confirmed by TEM analysis, and the typical optical properties were also confirmed, with the higher emission at $380 \mathrm{~nm}$ by using $\lambda_{\text {ex }} 325 \mathrm{~nm}$. The authors employed a synthetic covalent approach to obtain polyamide, polyurea-urethane and polyester materials, while a non-covalent approach to obtain polymethylmetacrylate. SEM and TEM analysis show the formation of fibers having different morphology, depending by the amount of CNPs in the composite material. These new materials have been used as photocatalysts for the (i) photoreduction of silver ion to nanoparticles; (ii) the photooxidation of benzylalcohol to the benzaldehyde and (iii) the photogeneration of $\mathrm{H}_{2}$. In particular, the

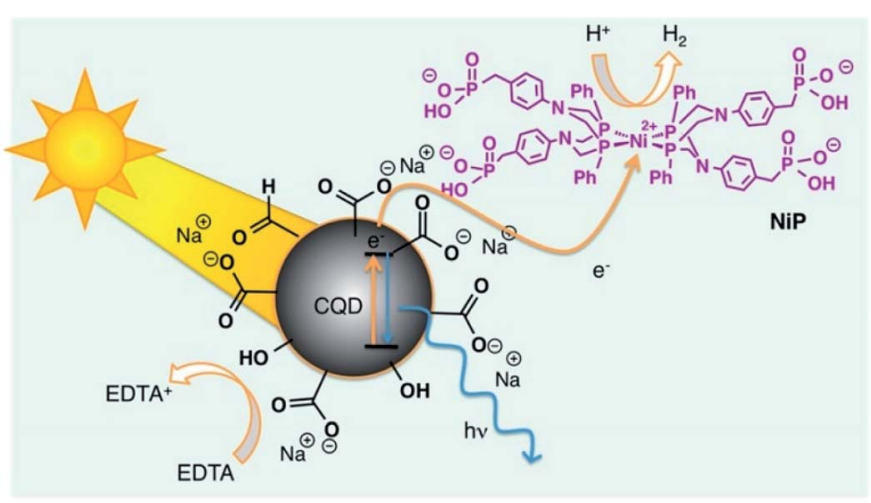

Fig. 2 Representation of solar $\mathrm{H}_{2}$ production using the hybrid CNPs (reproduced from ref. 15, Copyright 2019 American Chemical Society). 
photogeneration of $\mathrm{H}_{2}$ has been employed by using polyesteramide with N-doped CNPs. The optical properties of CNPs embedded into polymer matrix are different respect to the free nanoparticles (the emission maximum in fact is shifted up to $100 \mathrm{~nm}$ ). Reaction between benzyl alcohol, polyester-amide, Ndoped CNPs and hydrogen peroxide under irradiation at $405 \mathrm{~nm}$, leads to benzaldehyde with the formation of $\mathrm{H}_{2}$. The possibility to irradiate the composite nanocatalyst at $405 \mathrm{~nm}$ allows to avoid the photolysis of hydrogen peroxide, which undergoes by using excitation wavelength of $365 \mathrm{~nm}$ or shorter.

MacFarlane and co-workers used CNPs to prepare a nanocatalyst for the ring opening of epoxides (Scheme 2), starting from primary alcohols, by using visible-light irradiation at room temperature and methanol as solvent. ${ }^{17}$ They functionalized the external shell of the nanoparticles with hydrogen sulphate groups, leading to the possibility to photogenerate acidity after the exposure to the visible light. The obtained CNPs are monodisperse, with a size in the range of 2-9 $\mathrm{nm}$. These synthesized systems exhibit a light-switchable acidity thanks to photoexcitation and charge separation of the nanoparticles, that as a consequence carry the acid groups to an electron withdrawing effect. This nanosystem is recyclable with no loss of efficiency. The authors compared the sulphate functionalized CNPs with graphene oxide and with pristine CNPs, showing that in all cases conversion values are higher by using the sulphate functionalized CNPs. Notably, the photoirradiation of CNPs leads to an increase of the reactivity, due to an additional proton release from the sulphate groups, thus obtaining a stronger acid catalysis.

Das and co-workers used CNPs in a water soluble polymer matrix (polyurethane) to catalyse the one-pot regioselective hydroxylation of aromatic compounds, by using hydrogen peroxide and UV-light (Scheme 3$).{ }^{18}$ Reaction does not occur in the absence of the nanocatalyst, while in the presence of $5 \% \mathrm{wt}$ of the CNPs-polyurethane, after $1 \mathrm{~h} 98 \%$ of yield has been observed. The authors tested also the catalysis in the presence of the polymer matrix only, without nanoparticles. In this case, after $3 \mathrm{~h}$ at $50{ }^{\circ} \mathrm{C}$, only $13 \%$ of yield was obtained. These results highlight the role of CNPs in the photocatalytic hydroxylation. This catalyst can be saved and be re-used showing no loss in performance. Although the mechanism needs to be clarify, the authors proposed a radical pathway with the CNPs as electron reservoir.

\section{Acid-base catalysts}

Willner and co-workers reported on $\mathrm{Cu}^{2+}$-CNPs functionalized with $\beta$-cyclodextrin and $\mathrm{Cu}^{2+}$-modified carbon nitride nanoparticles and tested their catalytic activity in the horseradish peroxidase-mimicking. ${ }^{19}$ Both catalysts catalyse the oxidation of

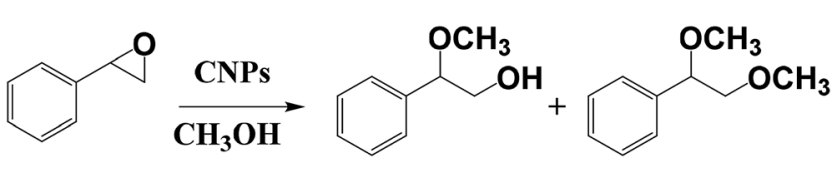

Scheme 2 Ring opening reaction catalyzed by CNPs.

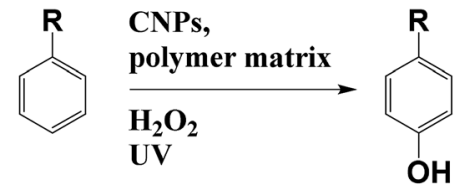

$\mathrm{R}=-\mathrm{COOH},-\mathrm{CHO},-\mathrm{NO}_{2},-\mathrm{CH}_{3},-\mathrm{CN}$

Scheme 3 Hydroxylation of aromatic compounds catalysed by CNPS in polymer matrix and hydrogen peroxide.

dopamine to aminochrome, by using $\mathrm{H}_{2} \mathrm{O}_{2}$ as oxidant (Scheme 4). Control experiments show that $\mathrm{Cu}^{2+}$ ions alone or pristine CNPs did not catalyze the oxidation. Similarly, the presence of $\beta$-cyclodextrin increase the oxidation rate of $c a$. 4 -fold respect to the unfunctionalized nanoparticles. This enhancement of the reaction rate is probably due to the inclusion of dopamine into the $\beta$-cyclodextrin, thus leading to an increase of the local concentration of the substrate. Notably, these catalysts show the possibility of being functionalized, which makes them also suitable as sensor for hydrogen peroxide.

Recently, Farhadian and co-workers reported on CNPs able to catalyse the oxidation of phenols by using hydrogen peroxide as oxidant. $^{20}$ In particular, they obtained a N-doped CNPs starting from biopolymers (gum tragacanth and chitosan) by hydrothermal carbonization of the starting material. SEM analysis show higher sizes respect to the common carbon nanoparticles (65 $\mathrm{nm}$ for CNPs-gum tragacanth and 40 for chitosan-CNPs), monodispersed and with a spherical shape. They demonstrated the ability as catalysts in the oxidation of phenol via radical mechanism by using $\mathrm{H}_{2} \mathrm{O}_{2}$. In 20 minutes, this catalyst shows a 99\% phenol degradation, exhibiting good recyclability. Control experiments show that only the presence of nanocatalysts and hydrogen peroxide together leads to the phenol degradation.

Recently, our research group developed a "new derivatization protocol" of CNPs based on the covalent functionalization of the external shell, leading to a stable catalyst. ${ }^{21}$ In particular, we anchored a chiral Mn-salen catalyst onto carbon nanoparticles for the enantioselective epoxidation of some selected alkenes (dihydronaphtalene, $\mathrm{CN}$-chromene and cis- $\beta$-ethylstyrene, Scheme 5). Morphology of the nanocatalyst has been elucidated by XPS, SEM and TEM analysis. In particular, XPS measurements confirmed the covalent linkage between CNPs and the chiral catalyst, suggesting an average coverage of $33 \%$ by the

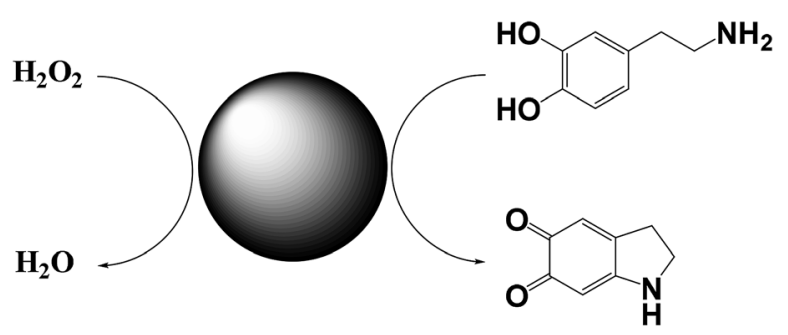

Scheme 4 Conversion of dopamine into aminochrome by using $\mathrm{Cu}^{2+}-$ CNPs functionalized with $\beta$-cyclodextrin. 


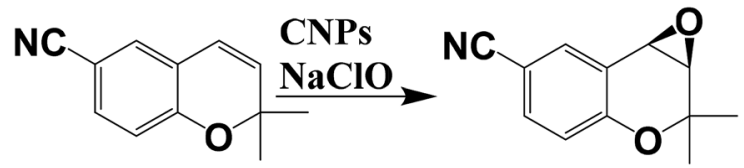

Scheme 5 Enantioselective epoxidation of $\mathrm{CN}$-chromene by using chiral CNPs.

chiral Mn-salen catalyst, with a diameter of the nanoparticles of ca. $8 \mathrm{~nm}$. Epoxidation reactions were carried out by using $\mathrm{NaClO}$ as oxidant in dichloromethane, showing similar enantiomeric excesses respect to the control experiments for dihydronaphtalene and $c i s-\beta$-ethylstyrene. Otherwise, in the case of $\mathrm{CN}$-chromene, the enantiomeric excesses measured with the nanocatalysts were higher respect to the single-molecules catalyst, highlighting the role of the nanoparticle in the improvement of the enantioselectivity.

\section{Electro catalysts}

Mixed nanostructures, obtained by hydrothermal method mixing CNPs with transition metal oxides, such as $\mathrm{SnO}_{2}$ and $\mathrm{Co}_{3} \mathrm{O}_{4}$, were reported by Kang and co-workers. ${ }^{22}$ The starting CNPs are monodispersed, with a size diameter of $5.32 \mathrm{~nm}$. HR-TEM images show a crystal lattice spacing of $c a .0 .320 \mathrm{~nm}$, indicating a (002) lattice planes of graphitic carbon. After the mixing with the metal oxides, TEM analysis shows lamellar-like structures. XPS and XRD measurements confirmed the presence of the metal atoms in the hybrid nanostructure.

These CNPs $/ \mathrm{SnO}_{2}-\mathrm{Co}_{3} \mathrm{O}_{4}$ are able to electro-catalyze water oxidation and the oxygen evolution reaction (Fig. 3). Different molar ratios of $\mathrm{Sn}$ : Co have been tested, finding the best performance with a $1: 3$ ratio. They proved that the active centre of these nanocomposites are the Co atoms and that the $\mathrm{SnO}_{2}$ only improves electronic conductivity. In order to demonstrate the positive role of CNPs in the water oxidation, the authors tested the presence of three different size of carbon nanoparticles (5, 10 and $100 \mathrm{~nm}$, respectively). The higher electrocatalytic activities has been found for nanoparticles with $5 \mathrm{~nm}$ of diameter, also compared with the system without the presence of CNPs. In particular, this study demonstrates how the organic nanostructured material acts as shield, protecting

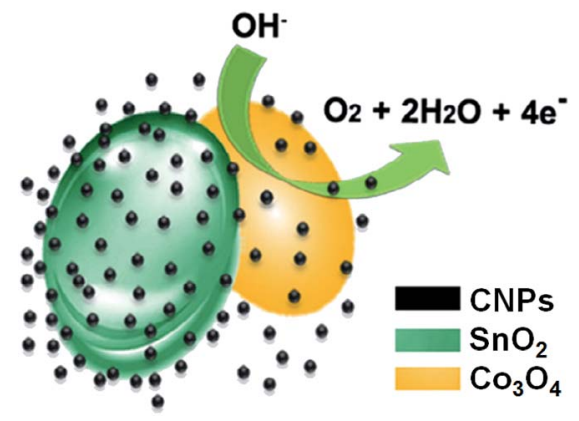

Fig. 3 Electrocatalytic water oxidation catalysed by $\mathrm{CNPs} / \mathrm{SnO}_{2} /$ $\mathrm{CO}_{3} \mathrm{O}_{4}$ (adapted from ref. 22, with permission of Elsevier Ltd.). the Co metal ions, leading to a nanocatalyst with high activity and stability, in particular in alkaline medium.

Jin and co-workers have been used CNPs also in the $\mathrm{Li}-\mathrm{CO}_{2}$ batteries, to reduce the charge overpotential, leading longer life time. ${ }^{23}$ They prepared a composite of CNPs and graphene nanosheets, having a high number of edge defects near the carbon nanoparticles, and holes near the graphene units. TEM analysis reveals monodispersed nanoparticles size in the range of 5-10 nm, with a lattice spacing of $3.5 \AA$, corresponding to the (002) plane. FT-IR analysis shows decrease of intensity and a shift of some peaks, suggesting a $\pi-\pi$ stacking interactions between CNPs and graphene nanosheets. In particular, the authors optimized the CNPs/graphene ratio, in order to obtain the higher electrocatalytic activity for the $\mathrm{CO}_{2}$ reduction. The best results were obtained by using $0.3 \mathrm{CNPs} /$ graphene ratio, also if compared to only graphene. The energy efficiency and long-term stability of the adduct CNPs/graphene was also confirmed in $\mathrm{Li}-\mathrm{CO}_{2}$ batteries, demonstrating that the discharge voltages of system containing the CNPs/graphene adduct are higher than those of systems containing only graphene. This higher efficiency is due to the morphology of the adduct. The edge defects near the carbon nanoparticles act as catalytic sites during the cycle charge/discharge of the battery, and the holes allow the diffusion of the carbon dioxide and ions. The use of this catalyst as the cathode in $\mathrm{Li}-\mathrm{CO}_{2}$ batteries helps to support the synthesis and degradation of $\mathrm{Li}_{2} \mathrm{CO}_{3}$, increasing the cycle number up to 235 .

Yang and co-workers used a sandwich-structured of PtCographene/CNPs/graphene as catalyst (Fig. 4), obtained by electrodeposition, in the methanol oxidation. ${ }^{24}$ The carbon dots, obtained by thermal decomposition of organic material, show an average size of $3.2 \mathrm{~nm}$ and contain $-\mathrm{OH}$ and $-\mathrm{COOH}$ groups on their surface. Optical properties are typical of CNPs, in particular fluorescence intensity shows the highest value at $424 \mathrm{~nm}$ by using excitation wavelength of $350 \mathrm{~nm}$.

TEM and SEM analysis show that PtCo nanoparticles are uniformly dispersed between graphene sheets due to the presence of CNPs, which prevent the aggregation of graphene sheets and leads to higher binding sites for PtCo nanoparticles to the graphene layers.

The system containing CNPs shows superior stability and catalytic activity compared to PtCo/graphene only, thanks to the
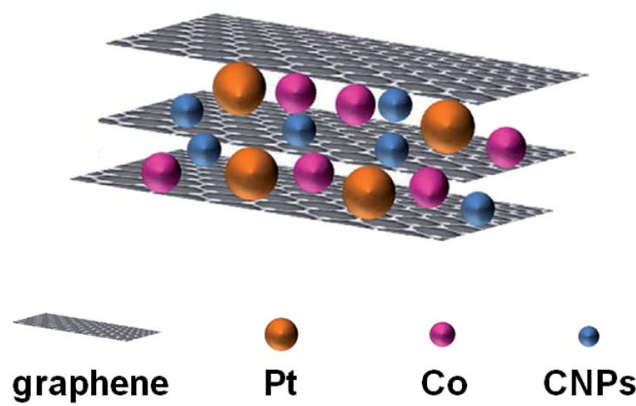

Fig. 4 Schematic representation of the catalyst used in the methanol oxidation (adapted from ref. 24, with permission of Elsevier). 
inclusion of CNPs into the graphene sheets. The presence of CNPs increase: (i) the electron conductivity between graphene layers, (ii) the dispersion of PtCo nanoparticles, (iii) the mutual effect of Pt and Co nanoparticles. In addition, the $-\mathrm{OH}$ and $-\mathrm{COOH}$ groups on the CNPs surface increase the reactivity because they consume intermediate species, like CO.

Zhang and co-workers synthesized, by hydrothermal decomposition, a carbon-based catalyst based on nitrogen and sulfur co-doped CNPs and reduced graphene oxide (N,S-CNPs/ rGO). ${ }^{25}$ Morphology was elucidated by TEM analysis, showing an average size of $c a .2 \mathrm{~nm}$. TEM images of the composite between CNPs and graphene show an uniform coverage of the graphene surface by the nanoparticles, with wrinkled and rougher surfaces. Chemical characterization was performed by FTIR and XPS, founding the presence of hydroxyl, carbonyl and epoxy oxygen groups, as well as the presence of nitrogen and sulfur atoms.

The adduct N,S-CNPs/rGO was employed as electrocatalysts in the oxygen reduction reactions. In particular, the authors demonstrated that the CNPs/rGO samples are better in comparison to the doped rGO only, due to the copious edges and active sites on carbon nanoparticles, and that both $\mathrm{N}$ and $\mathrm{S}$ co-doping and substrate structure are essential for the catalytic activity (Fig. 5).

Also research group of Prof. Ghilane used CNPs as catalysts for the oxygen reduction process. ${ }^{26}$ In particular, N-doped CNPs (N-CNP) were synthesized by microwave irradiation, and a polyimidazolium ionic liquid polymer (ILP) has been employed as host for these nanoparticles.

Chemical composition of the composite was elucidated by XPS measurements, and size distribution, in a range of 7$16 \mathrm{~nm}$, has been determined by DLS (Dynamic Light Scattering) and TEM images. A comparison between the activity of the composite material (N-CNPs/ILP) and the control system ( $\mathrm{N}$ CNPs) shows higher activity of N-CNPs/ILP in the oxygen

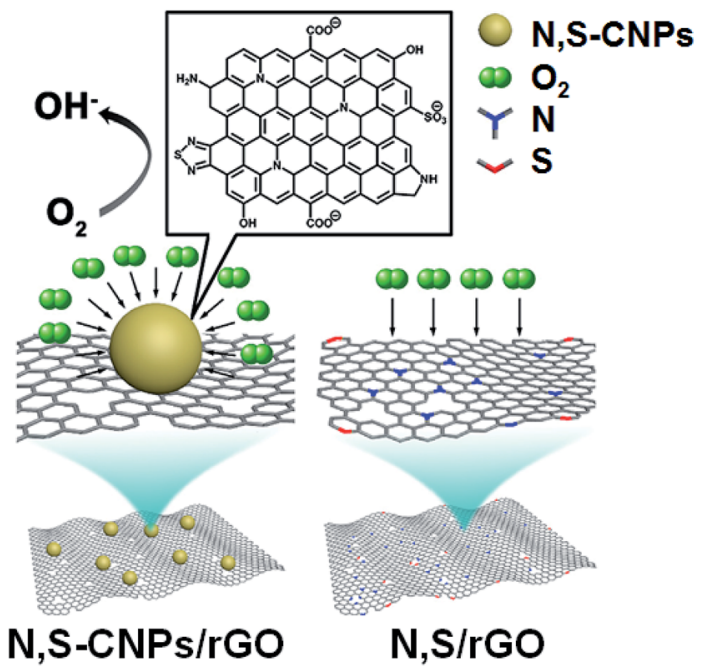

Fig. 5 Schematic representation of the catalyst for the oxygen reduction reactions (adapted from ref. 25, with permission of Elsevier). reduction reaction, as demonstrated by the hydrogen peroxide variation, with a selectivity of $97 \%$ for reducing oxygen to water.

The high performance of this kind of catalyst in oxygen reduction reactions is due to the interaction between the ionic liquid and the CNPs, probably due to the presence of electrostatic interactions.

\section{Conclusions and future perspectives}

Until today, the nanocatalysis has been dominated by metal nanoparticles. Only recently, the wide diffusion of carbon nanoparticles allows to apply this new nanomaterials also in the catalytic field. The examples reported in this minireview point out the possibility to use carbon nanoparticles also as catalysts. In particular, as here reported, different reactions can be catalysed by the appropriate carbon nanocatalyst. Due to the high biocompatibility, low cost, optical properties and relative easy functionalization of the external shell, these nanoparticles could be the "next generation" of nanocatalysts. In particular, an ideal catalyst should be unlimitedly recoverable, thus implying high chemical, physical and thermal stability. In this context, CNPs represent excellent candidates, due to their high stability in extreme chemical and thermal conditions.

Furthermore, the possibility to anchor onto their surface "theoretically" any organic molecule or organic/inorganic catalyst paves the way to the obtaining of catalysts for a wide reaction classes.

If compared to other nanoparticle classes, such as gold nanoparticles, starting material (carbon source) is very low cost and the preparation of pristine CNPs does not require expensive synthesis.

The peculiar optical properties, as previously reported, allow to the realization of versatile "antenna-systems", able to absorb a very wide range of wavelengths, thus collecting high amounts of energy for several applications in, but not only, photocatalysis.

In addition, these nanoparticles can be solubilized in a wide range of solvent, depending by the nature of the external shell (hydrophobic or hydrophilic), thus increasing the number of application fields.

\section{Conflicts of interest}

There are no conflicts to declare.

\section{Acknowledgements}

The authors thank the University of Catania (Piano della Ricerca di Ateneo 2016-2018) for financial support.

\section{Notes and references}

1 V. Vakhrushev, V. I. Kodolov, A. K. Haghi and S. C. Ameta, Carbon Nanotubes and Nanoparticles: Current and Potential Applications, CRC Press, Taylor \& Francis Group, 1st edn, 2019. 
2 Y. Wang, Y. Zhu, S. Yu and C. Jiang, RSC Adv., 2017, 7, 4097340989.

3 P. Zhao and L. Zhu, Chem. Commun., 2018, 54, 5401-5406.

4 W. Liu, C. Li, Y. Ren, X. Sun, W. Pan, Y. Li, J. Wang and W. Wang, J. Mater. Chem. B, 2016, 4, 5772-5788.

5 S. N. Qu, H. Chen, X. M. Zheng, J. S. Cao and X. Y. Liu, Nanoscale, 2013, 5, 5514-5518.

6 A. W. Zhu, Q. Qu, X. L. Shao, B. Kong and Y. Tian, Angew. Chem., Int. Ed., 2012, 51, 7185-7189.

7 N. Licciardello, S. Hunoldt, R. Bergmann, G. Singh, C. Mamat, A. Faramus, J. L. Z. Ddungu, S. Silvestrini, M. Maggini, L. De Cola and H. Stephan, Nanoscale, 2018, 10, 9880-9891.

8 H. Wang, G. Cao, Z. Gai, K. Hong, P. Banerjee and S. Zhou, Nanoscale, 2015, 7, 7885-7895.

9 N. Tuccitto, G. Li-Destri, G. M. L. Messina and G. Marletta, J. Phys. Chem. Lett., 2017, 8, 3861-3866.

10 G. Li-Destri, L. Fichera, A. Zammataro, G. Trusso Sfrazzetto and N. Tuccitto, Nanoscale, 2019, 11, 14203-14209.

11 V. La Paglia Fragola, F. Lupo, A. Pappalardo, G. Trusso Sfrazzetto, R. M. Toscano, F. P. Ballistreri, G. A. Tomaselli and A. Gulino, J. Mater. Chem., 2012, 22, 20561-20565.

12 A. D'Urso, C. Tudisco, F. P. Ballistreri, G. G. Condorelli, R. Randazzo, G. A. Tomaselli, R. M. Toscano, G. Trusso Sfrazzetto and A. Pappalardo, Chem. Commun., 2014, 50, 4993-4996.

13 G. Trusso Sfrazzetto, S. Millesi, A. Pappalardo, R. M. Toscano, F. P. Ballistreri, G. A. Tomaselli and A. Gulino, Catal. Sci. Technol., 2015, 5, 673-679.
14 M. Muthulingam, I.-H. Lee and P. Uthirakumar, J. Colloid Interface Sci., 2015, 455, 101-109.

15 B. C. M. Martindale, G. A. M. Hutton, C. A. Caputo and E. Reisner, J. Am. Chem. Soc., 2015, 137, 6018-6025.

16 D. Mosconi, D. Mazzier, S. Silvestrini, A. Privitera, C. Marega, L. Franco and A. Moretto, ACS Nano, 2015, 9, 4156-4164.

17 H. Li, C. Sun, M. Ali, F. Zhou, X. Zhang and D. R. MacFarlane, Angew. Chem., Int. Ed., 2015, 54, 8420-8424.

18 V. K. Das, S. Gogoi, B. M. Choudary and N. Karak, Green Chem., 2017, 19, 4278-4283.

19 M. Vázquez-González, W.-C. Liao, R. Cazelles, S. Wang, X. Yu, V. Gutkin and I. Willner, ACS Nano, 2017, 11, 32473253.

20 M. Pirsaheb, S. Moradi, M. Shahlaei and N. Farhadian, J. Hazard. Mater., 2018, 353, 444-453.

21 A. Zammataro, C. M. A. Gangemi, A. Pappalardo, R. M. Toscano, R. Puglisi, G. Nicotra, M. E. Fragalà, N. Tuccitto and G. Trusso Sfrazzetto, Chem. Commun., 2019, 55, 5255-5258.

22 S. Zhao, C. Li, J. Liu, N. Liu, S. Qiao, Y. Han, H. Huang, Y. Liu and Z. Kang, Carbon, 2015, 92, 64-73.

23 Y. Jin, C. Hu, Q. Dai, Y. Xiao, Y. Lin, J. W. Connell, F. Chen and L. Dai, Adv. Funct. Mater., 2018, 28, 1804630.

24 S. Yang, F. Zhang, C. Gao, J. Xia, L. Lu and Z. Wang, J. Electroanal. Chem., 2017, 802, 27-32.

25 P. Zhang, J.-S. Wei, X.-B. Chen and H.-M. Xiong, J. Colloid Interface Sci., 2019, 537, 716-724.

26 T.-N. Pham-Truong, C. Ranjan, H. Randriamahazaka and J. Ghilane, Catal. Today, 2019, 335, 381-387. 